\title{
Systematic Results on Reactions and Scattering with Weakly Bound Nuclei at Near barrier Energies
}

\author{
P.R.S. Gomes ${ }^{1}$, J. Lubian ${ }^{1}$ and L.F. Canto ${ }^{2}$ \\ ${ }^{1}$ Instituto de Física, Universidade Federal Fluminense, Av. Litoranea s/n, 24210-340 Niteroi, R.J. , Brazil \\ ${ }^{2}$ Instituto de Física, Universidade Federal do Rio de Janeiro, CP 68528, Rio de Janeiro, R.J., 21941-972, Brazil
}

\begin{abstract}
We present a systematic interpretation of the results available in literature on the complete fusion, total fusion and total reaction cross sections induced by weakly bound nuclei and compare the results with those induced by tightly bound nuclei. We also investigate the behaviour of the energy dependence of the optical potential at near barrier energies for the same systems. We discuss the results of the systematic and present some experimental challenges on the future of this subject.
\end{abstract}

\section{Introduction}

In the last two decades, large efforts have been made to understand the role of the breakup of weakly bound nuclei on different reaction and scattering processes [1].

Examples of usual questions not yet fully answered are: Does the breakup enhances or suppress fusion cross sections? Is the effect on complete fusion or total fusion? Is the effect the same at energies above and below the Coulomb barrier? How does this effect change with the target mass? Does the breakup affect the elastic scattering and the usual threshold anomaly of the optical potential at near barrier energies? Do different breakup threshold energies affect the total reaction cross section significantly?

We will try to answer partially some of those questions in the following, based on the systematic results available in literature.

\section{Effect of low binding energy and breakup on the fusion cross section}

We start this section by saying that the effects that we investigate, contrary to what one expected twenty years ago, are not so remarkable as the huge sub-barrier fusion enhancement observed for highly deformed nuclei, when compared with predictions from one dimensional barrier penetration models. Another initial and fundamental point to be raised is that when one talks about enhancement or hindrance of fusion, one must be very clear in relation to what one is talking about.

There are two main approaches to investigate this subject. The first is to compare fusion data with predictions from some theory. The difference between them, $\Delta \sigma_{\mathrm{F}}$, is attributed to the ingredients missing in the theory. The second is to compare fusion data for different systems, including tightly bound ones.

There are two kinds of effects to be investigated. The first is static effects, caused by the longer tail of the optical potential, owing to the low binding energies of the weakly bound and specially halo nuclei. This effect gives rise to lower and thicker barriers when compared with tightly bound systems, and enhances fusion cross section at sub-barrier energies not too much below the barrier. The second kind of effect is the dynamic, which is due to the strong coupling between the elastic channel and the continuum states representing the breakup channel. These effects have been explained in details by Canto et al. $[2,3]$. When one compares data with theory, the comparison may be (i) with single channel with standard densities of the nuclei $\left(\Delta \sigma_{\mathrm{F}}\right.$ is due to static + dynamic effects); (ii) single channel with realistic densities $\left(\Delta \sigma_{\mathrm{F}}\right.$ comes from all channels but the static effect is already taken into account); (iii) coupled channel calculations taking into account all bound channels $\left(\Delta \sigma_{\mathrm{F}}\right.$ comes from the coupling to the continuum); (iv) Continuum Discretized Coupled Channel (CDCC) calculations $\left(\Delta \sigma_{\mathrm{F}}\right.$ should vanish). The comparison is, for sure, strongly model dependent. This may lead to controversies like the apparent one for fusion of ${ }^{6} \mathrm{He}+{ }^{209} \mathrm{Bi}[4]$ and ${ }^{6} \mathrm{He}+{ }^{238} \mathrm{U}$ [5] data. Recently it has been clearly shown [6] that there are no incompatibility between the two sets of data, which were originally analyzed using different models. We believe that the most interesting investigation is on the dynamic effects of the breakup on fusion.

If one wants to investigate a systematic behaviour of the fusion cross section for several systems, with different mass, charges and barrier parameters, data should be reduced in order to eliminate the static effects. It has been shown $[2,3]$ that the traditional reduction 
procedures of dividing the cross section by $\pi \mathrm{R}_{\mathrm{B}}{ }^{2}$ and the center of mass energy by $V_{B}$ do not work. Instead, a fusion function $\mathrm{F}_{\mathrm{exp}}=2 \mathrm{E} \sigma_{\mathrm{F}} /\left(\hbar \omega \mathrm{R}_{\mathrm{B}}{ }^{2}\right)$ should be plotted against the quantity $x=\left(\mathrm{E}-\mathrm{V}_{\mathrm{B}}\right) / \hbar \omega$. As one wants to eliminate the effect of all inelastic channels in the present investigation, it is necessary to renormalize $\mathrm{F}_{\exp }$ to $\overline{F_{\exp }}$ $=\mathrm{F}_{\exp }\left(\sigma_{\mathrm{F}}{ }^{\mathrm{W}} / \sigma_{\mathrm{F}}{ }^{\mathrm{CC}}\right)$, where $\sigma_{\mathrm{F}}{ }^{\mathrm{W}}$ is the fusion cross section calculated by the Wong approximation and $\sigma_{\mathrm{F}}{ }^{\mathrm{CC}}$ is the cross section obtained with a reliable bare potential and coupled channel calculations including all bound excited channels. $\overline{F_{\text {exp }}}$ is then compared with a Universal Fusion Function (UFF), $\mathrm{F}_{0}(\mathrm{x})=\ln [1+\exp (2 \pi x)]$. It has been shown [2,3] that for any tightly bound systems for which only inelastic channels are important in the coupling scheme (tens of systems were tested), $\overline{F_{\exp }}$ coincides with the UFF. Heavy and symmetric tightly bound systems are discussed by Canto [7]. For the weakly bound systems, the difference between $\overline{F_{\exp }}$ and the UFF is attributed to the coupling of breakup and transfer channels not included in the coupled channel calculations.

We investigated the behaviour of total fusion for around 20 light weakly bound systems, for ${ }^{6} \mathrm{Li},{ }^{7} \mathrm{Li},{ }^{9} \mathrm{Be}$ projectiles and targets from ${ }^{12} \mathrm{C}$ to ${ }^{64} \mathrm{Zn}$. For energies above the barrier, all of them, with a few exceptions, coincide with the UFF. There are few sub-barrier data for these systems, but the ones available also coincide with the UFF. In figure 1 we show a few of those systems, as examples. Data are from ref [8-13]. We believe that an old controversy about total fusion suppression above the barrier for light systems $[11,13,14]$ is over. For heavier targets, the renormalized experimental fusion functions coincide with the UFF above the barrier and some subbarrier fusion enhancement is observed. In figure 1 we show a few of those systems, as examples. For the complete fusion with heavy targets, there is around $30 \%$ suppression above the barrier, when compared with the UFF, and some enhancement at sub-barrier energies. We show in figure 2 a few systems of this kind, selected among all systems available in literature. Data are from ref $[10,15-18]$. So far, there is no systematic for the complete fusion suppression as a function of the charge or mass of the targets. This is due to the difficulty in separating events from complete fusion and incomplete fusion for light systems, since most of their evaporation residues coincide. It is an experimental challenge to separate complete and incomplete fusion for light systems. Another experimental challenge is to separate total fusion events from possible transfer events. If one is not able to do so, only total fusion + transfer cross sections are measured. As one observes suppression of complete fusion above the barrier and no effect on total fusion at the same energy range, one attributes the suppression of complete fusion to the presence of incomplete fusion. Very few systems, for both tightly bound and stable weakly bound, do not follow the systematic found for tens of systems. We believe that there might be three possible explanations for the exceptions: (i) a very special feature of that particular system; (ii) problems with the data; (iii) wrong or incomplete coupled channel calculations or bare potential used. We believe that one does not need more data of total fusion above the barrier for other similar systems, unless the possible transfer process is clearly separated from total fusion.

We show in figure 3 the results for total fusion of halo nuclei available in literature. ${ }^{6} \mathrm{He},{ }^{8} \mathrm{He}$ and ${ }^{11} \mathrm{Be}$

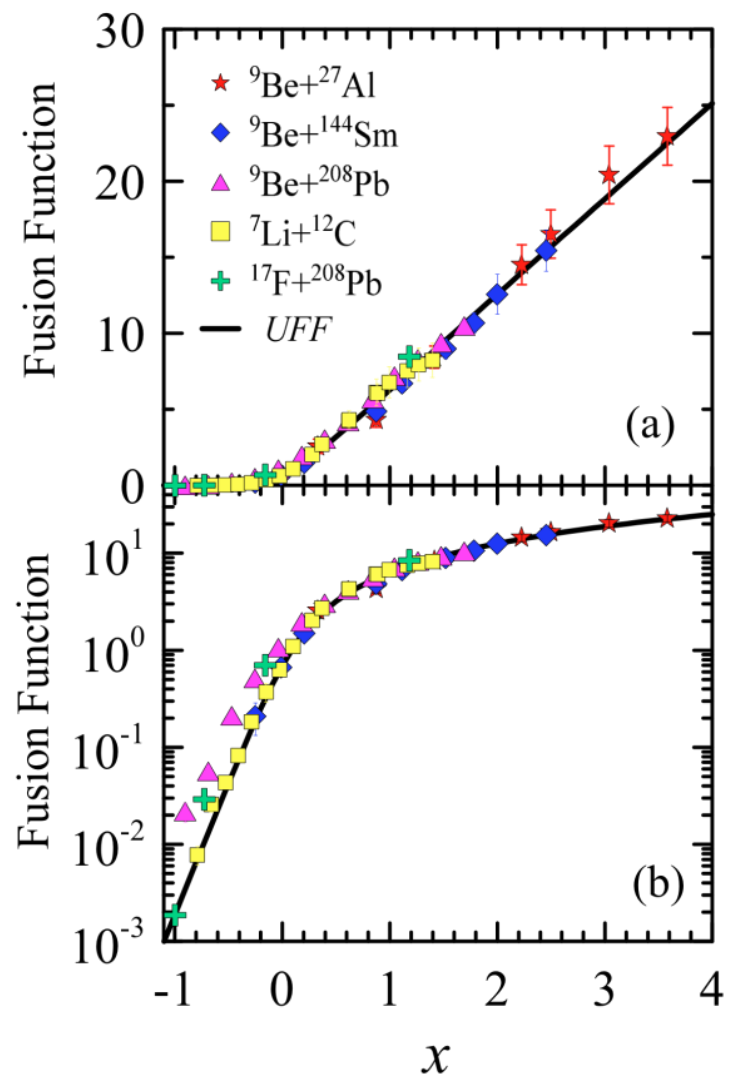

Fig. 1. Renormalized fusion function for total fusion of several stable weakly bound systems. The curve is the UFF. See text for details. The linear plot is better to observe the effects above the Coulomb barrier whereas the log plot is better to observe subbarrier effects.

projectiles were used on different targets [4,5,16, 19-23]. For most systems there are no data or few data at subbarrier energies. Although there is not a clear systematic as found for stable systems, one can observe a clear trend for around $30 \%$ fusion suppression above the barrier and some sub-barrier fusion enhancement. The ${ }^{6} \mathrm{He}+{ }^{64} \mathrm{Zn}$ system shows larger suppression whereas the ${ }^{6} \mathrm{He}+{ }^{197} \mathrm{Au}$ has larger fusion function than all the others. For ${ }^{11} \mathrm{Be}+$ ${ }^{209} \mathrm{Bi}$ there are two sets of data $[15,16]$, where the data with $(*)$ in fig 3 are those from ref [16]. The suppression of total fusion at energies above the barrier may be explained as due to the difficulty of the core to fuse (incomplete fusion) after the neutrons halo have been 


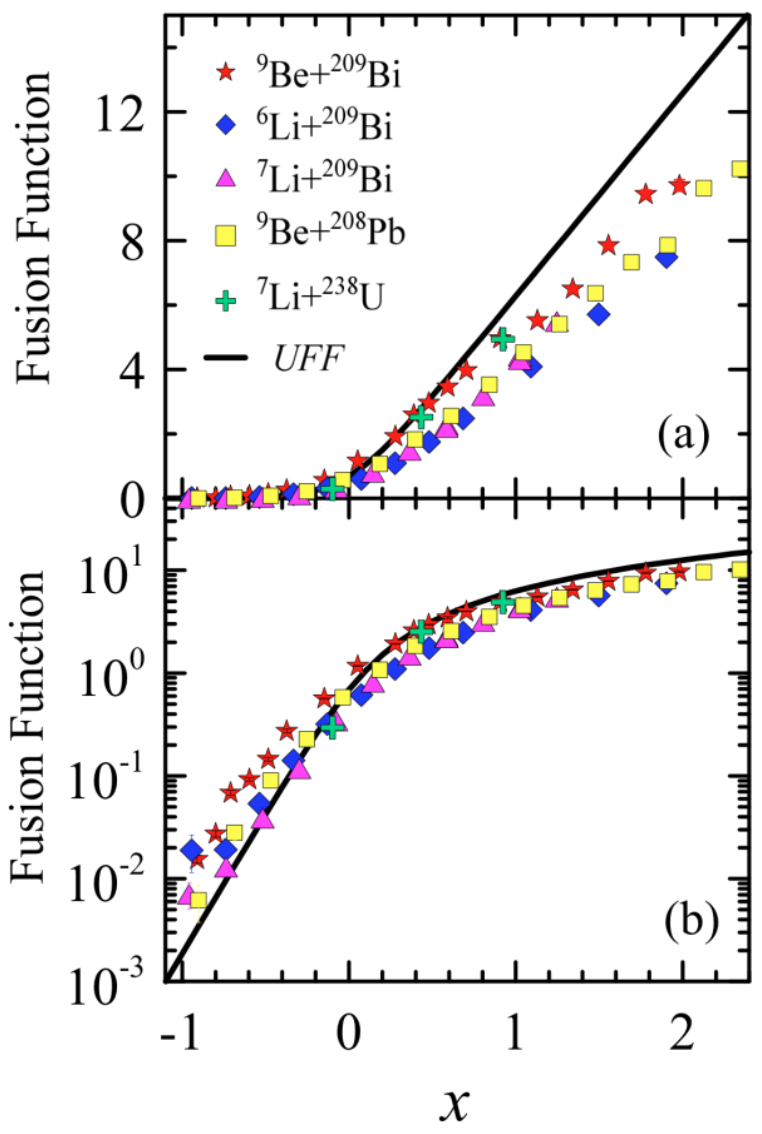

Fig. 2. Renormalized fusion functions for complete fusion of several stable weakly bound systems. The curve is the UFF. See text for details.

achieved a clearer systematic behaviour than at the moment. Experimental challenges are to obtain more data at sub-barrier energies and more precise data, with smaller error bars.

\section{Elastic scattering: breakup threshold anomaly and total reaction cross sections}

We investigate two aspects related with elastic scattering data: the presence or not of the usual threshold anomaly of the optical potential at near barrier energies and the systematic behaviour of the total reaction cross sections for different kinds of systems.

\subsection{The energy dependence of the optical potential}

For tightly bound systems, it is well known that at near barrier energies the optical potential presents an energy dependent behaviour known as threshold anomaly. As the energy decreases towards the barrier, reaction channels close and the imaginary potential decreases and vanishes.

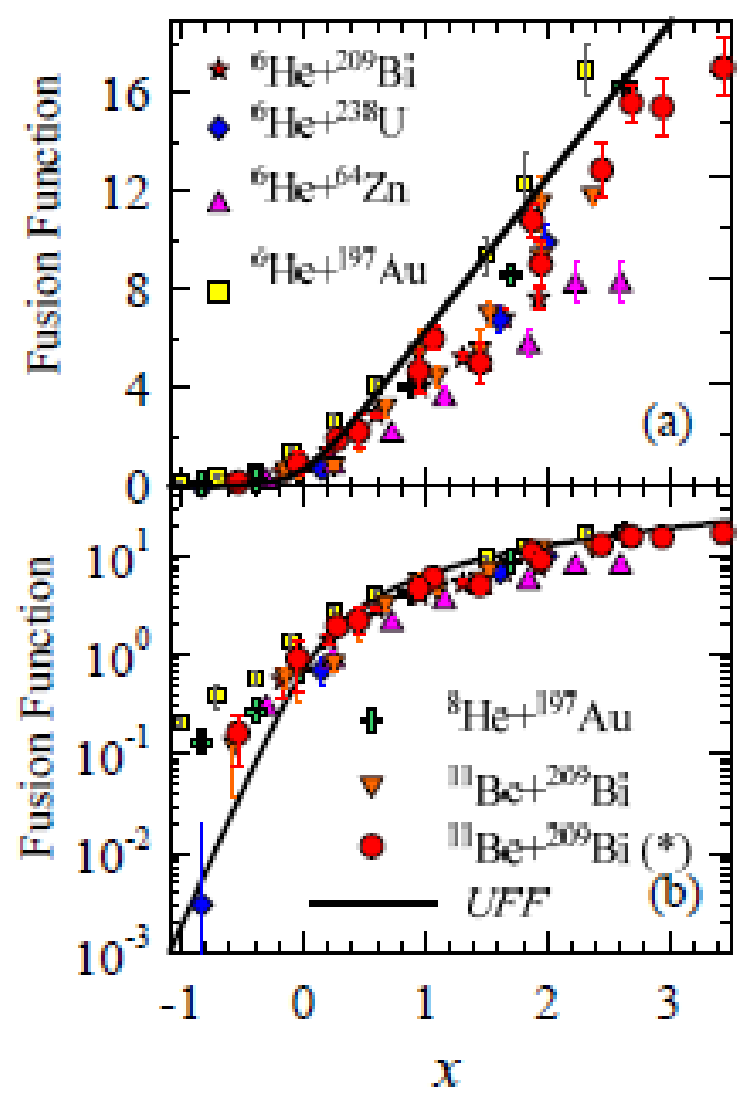

Fig. 3. Renormalized fusion functions for total fusion induced by neutron-halo nuclei. The curve is the UFF. See text for details.

Due to the dispersion relation, the real potential increases when the imaginary potential decreases. The attraction increases (attractive polarization potential) and consequently there is sub-barrier fusion enhancement. However, when weakly bound systems are involved, the behaviour may be different, because the breakup channel may be important even below the barrier. So, the imaginary potential does not decrease at the barrier energy. Indeed, it may increase. Consequently, the real potential decreases at this energy region and fusion is suppressed owing to this effect. This behaviour is called 'breakup threshold anomaly' (BTA) [24, 25].

Of course, the imaginary potential must decrease and vanish at lower energies. A detailed investigation of this effect is rather difficult because one needs precise elastic scattering data at sub-barrier energies, where the scattering is almost of Rutherford type and insensitive to the nuclear potential.

So far, at least 12 systems were investigated for the scattering of ${ }^{6} \mathrm{Li}$ and targets ranging from ${ }^{27} \mathrm{Al}$ to ${ }^{209} \mathrm{Bi}$. The BTA is found for all systems, mostly with the increase of the imaginary potential when energy decreases towards the barrier. There are very few systems for which there are conflicting analysis/results. For the scattering of the halo ${ }^{6} \mathrm{He}$ and ${ }^{8} \mathrm{~B}$ halo nuclei, the BTA is also clearly observed. For ${ }^{7} \mathrm{Li}$ scattering there are also several systems studied, but a systematic behaviour is not so clear, and the usual threshold anomaly is observed in 
some systems. The reason may be the larger threshold breakup energy for ${ }^{7} \mathrm{Li}$ and the competition between the repulsive polarization potential due to the breakup channel [26] and the attractive polarization potential due to the bound excited state of ${ }^{7} \mathrm{Li}$ [27]. So, the net breakup effect for ${ }^{7} \mathrm{Li}$ scattering is weaker than for ${ }^{6} \mathrm{Li}$. For the ${ }^{9} \mathrm{Be}$ scattering, there are not so many systems investigated, the behaviour is more similar to the ${ }^{7} \mathrm{Li}$ scattering and it is interesting to mention that the dispersion relation seems to be not satisfied for a few systems. For all those projectiles there are papers where there is a simultaneous fit of fusion and elastic scattering data and the optical potential is divided in a inner part connected with fusion and a direct part connected with superficial processes like breakup. It is then observed a threshold anomaly behaviour for the fusion potential and a BTA behaviour for the direct reaction potential (see, for example, ref. [28]). An experimental challenge is to measure very precise elastic angular distributions with small error bars and energies below the barrier, to investigate clearly the presence of the BTA and to determine the sub-barrier energy for which the imaginary potential vanishes. One work where this was done is ref [29] for the ${ }^{6} \mathrm{Li}+{ }^{144} \mathrm{Sm}$ scattering. One should avoid comparing the behaviour of different systems, with different Coulomb barriers, at the same near barrier energy, to avoid misleading conclusions.

\subsection{Systematic behaviour of total reaction cross sections}

There are tens of systems investigated, in more than 10 papers, related to the systematic behaviour of total reaction cross sections involving tightly bound, stable weakly bound and halo nuclei scattering. There are presently two reduction methods used to compare different systems in the same graph: the one proposed in 2005 by Gomes at al. [30] and the one proposed more recently by Shorto et al. [31], as an extension of the fusion function proposed by Canto et al. [2,3]. Both methods lead to qualitative similar results: the total reaction cross sections induced by halo nuclei are larger than for stable or non-halo weakly bound nuclei, which are similar or slightly larger than for tightly bound nuclei. The differences are more significant for heavy targets, due to the strong presence of the Coulomb breakup. Investigations should be done to decide which is the best reduction procedure to be used.

\section{Conclusions}

With the development of a method to compare different systems at the same plot and with the availability of several works on fusion and scattering of weakly bound nuclei, a partial systematic behaviour for the influence of breakup on the fusion and scattering has been reached. However, there are still many things to learn in this fascinating subject. We point out the need of more fusion data with radioactive and halo nuclei, specially at subbarrier energies and with better precision than the ones available in the present. Also, one needs the measurement of disentangled complete and incomplete fusion for light systems, in order to investigate the importance of incomplete fusion for those systems. It is also very important to disentangle transfer reactions from incomplete fusion and to measure the integrated cross sections of non-capture breakup for weakly bound systems, both above and below the barrier.

\section{Acknowledgements}

Te authors would like to thank the CNPq, FAPERJ and PRONEX for their partial financial support.

\section{References}

1. L.F. Canto et al., Phys. Rep. 424, 1 (2006).

2. L.F. Canto et al., J. Phys. G 36, 015109 (2009).

3. L. F. Canto et al., Nucl. Phys. A 821, 51 (2009).

4. J.J. Kolata et al., Phys. Rev. Lett. 81, 4580 (1998).

5. R. Raabe et al., Nature 431, 823 (2004).

6. P.R.S. Gomes et al., Phys. Lett. B 695, 320 (2011).

7. L.F. Canto - contribution to this proceedings.

8. G.V. Marti et al., Phys. Rev. C 71, 027602 (2005)

9. P.R.S. Gomes et al., Phys. Lett. B 634, 356 (2006) ; Phys. Rev. C 73, 064606 (2006)

10. M. Dasgupta et al., Phys. Rev. Lett. 82, 1395 (1999)

11. A. Mukherjee et al., Nucl. Phys. A 596, 299 (1996)

12. K.E. Rehm et al., Phys. Rev. Lett. 81, 3341 (1998)

13. A. Mukherjee et al., Phys. Lett. B 526, 295 (2002).

14. J. Takahashi et al., Phys. Rev. Lett. 78, 30 (1997).

15. C. Signorini et al., Eur. Phys. J. A 5, 7 (1999)

16. D.J. Hinde et al., Phys. Rev. C 81, 064611 (2010)

17. M. Dasgupta et al., Phys. Rev. C 70, 024606 (2004); Phys. Rev. C 66, 041602 (R) (2002)

18. R. Raabe et al., Phys. Rev. C 74, 044606 (2006)

19. A. Di Pietro et al., Phys. Rev. C 69, 044613 (2004).

20. C. Signorini et al., Nucl. Phys. A 735, 329 (2004).

21. A. Navin et al., Phys. Rev. C 70, 044601 (2004).

22. A. Lemasson et al., Phys. Rev. Lett. 103, 232701 (2010).

23. Yu. E. Penionzhkevich et al., Eur. Phys. J. A 31, 185 (2007).

24. P.R. S. Gomes et al., J. Phys. G 31, S1669 (2005).

25. M.S. Hussein et al., Phys. Rev. C 73, 044610 (2006).

26. J. Lubian et al., Nucl. Phys. A 791, 24 (2007).

27. J. Lubian et al., Phys. Rev. C 64, 027601 (2001).

28. A. Gomez Camacho et al., Phys. Rev. C 77, 054606 (2008).

29. J.M. Figueira et al., Phys. Rev. C 81, 024613 (2010).

30. P.R.S. Gomes et al., Phys. Rev. C 71, 017601(2005).

31. J.M. B. Shorto et al., Phys. Lett. B 678, 77 (2009). 